\title{
Assisted mails sorting and forwarding stands: performance analysis and ergonomic assessment
}

\author{
G.M. Acaccia*, M. Corvi ${ }^{\circ}$, W. Ferebauer*, R.C. Michelini* \\ *) Industrial Robot Design Research Group - University of Genova - Italy \\ \%) ELSAG SpA - Divisione Postale - Genova - Italy
}

\begin{abstract}
Industrial automation to-day covers broader areas, as robotic equipment provides technically sound solutions to remove on-process workers once taskcycle are properly assessed. Return on investment, however, remains sometimes open point and this is central question when competition is particularly strong. The postal services face all over the world drastic changes due to new means (information and communication technology) and business options (removal of governmental restriction on utilities), with enhanced attention on effectiveness by lean work-organisation, namely, mixed settings, where human operators are primary resource within sophisticated automatic processing lines. This is not without drawbacks, with implications on the plant ergonomics, and throughout productivity checks need be ran for actual operation lay-outs. The paper tackles such problems, exploiting simulation tools to accomplish beforehand analyses by virtual testing, in terms of resource allocation, process planning and labour protection.
\end{abstract}

Key words: Integrated design, Economics \& social impact and justification

\section{INTRODUCTION}

The exponential growth of information and communication technologies has drastically modified traditional activities, such as the mailing services, that happened to be consolidated as governmental branches or protected utilities. Now, the world-wide web supports an open space for information transmission and share, making unsound sheltered postal services, disjoined from duty-driven competitiveness, flexible specialisation and business integration. The full re-thinking of conventional mailing organisations goes

The original version of this chapter was revised: The copyright line was incorrect. This has been corrected. The Erratum to this chapter is available at DOI: 10.1007/978-0-387-35492-7_50 
through the identification of the main mission, by setting out the core business, up to the development of processing machines and related workcycle plans consistent with scope-oriented enterprises. These new horizons led to the special branch of postal automation, with relevant issues on facilities and techniques, to enhance the productivity with careful check of the economical return.

This branch quite soon represented a challenging domain, aiming, on one side, at intelligent automation set-ups (handwriting automatic reading units, etc.), the other side, at men-machine sustainable accountability (mixed human- and robot-attended work-lines, etc.). Technology-driven goals, thereafter, drastically modify postal organisations, with special requests about efficiency, as National borders do not turn down any more outsiders and world-wide-web alternatives can cover a large extent of communication needs; struggle to survive would follow on condition to carefully balance technology innovation against investment leanness. The choice of effective set-ups requires the accurate foresight of the life-cycle performance for actual work frames; a goal efficiently achieved by proper resort to CADbased digital mock-up and virtual reality testing. In the paper, these aspects of the design and development process are summarised, looking after an example case.

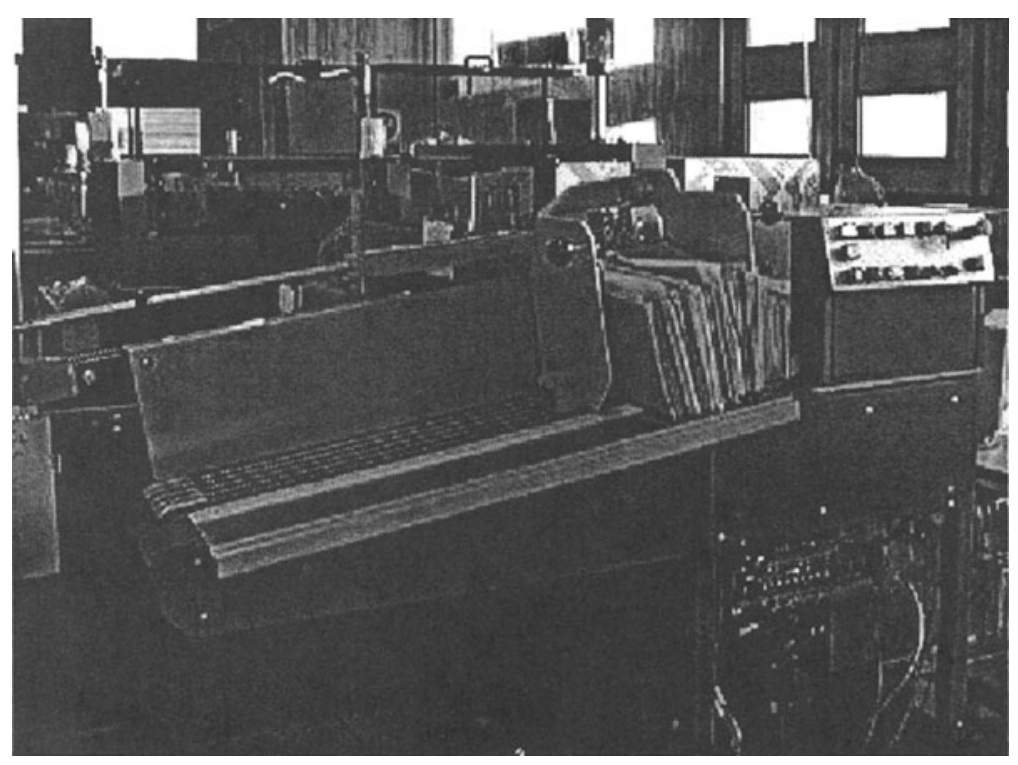

Figure 1. Typical in-feed magazine 


\section{AN EXAMPLE MAIL PROCESSING STAND}

Universal postal services face challenging tasks to compete within sectional classes (as for handled objects, conveying means, receiving points, etc.), but benefit from good advance with the processing of "flat" items of varying size and weigh, Fig. 1, presenting widely scattered addressees. Thus, restricting the present analysis to the generic "flats" class, an effective set-up would typically include automatic and manually operated units, to join high flexibility and productivity. The outfit should be tailored to the identified duty requirements and suitably built as a modular set-up, easily down- or upgraded as the case arises. Basically, we might distinguish:

- an input section, with items supplying stations and a set of automatic either manual in-feed modules;

- a processing section, with items recognition and coding modules, followed by dispatching and sorting equipment;

- an output section, with items routing and piling up into labelled assemblies.

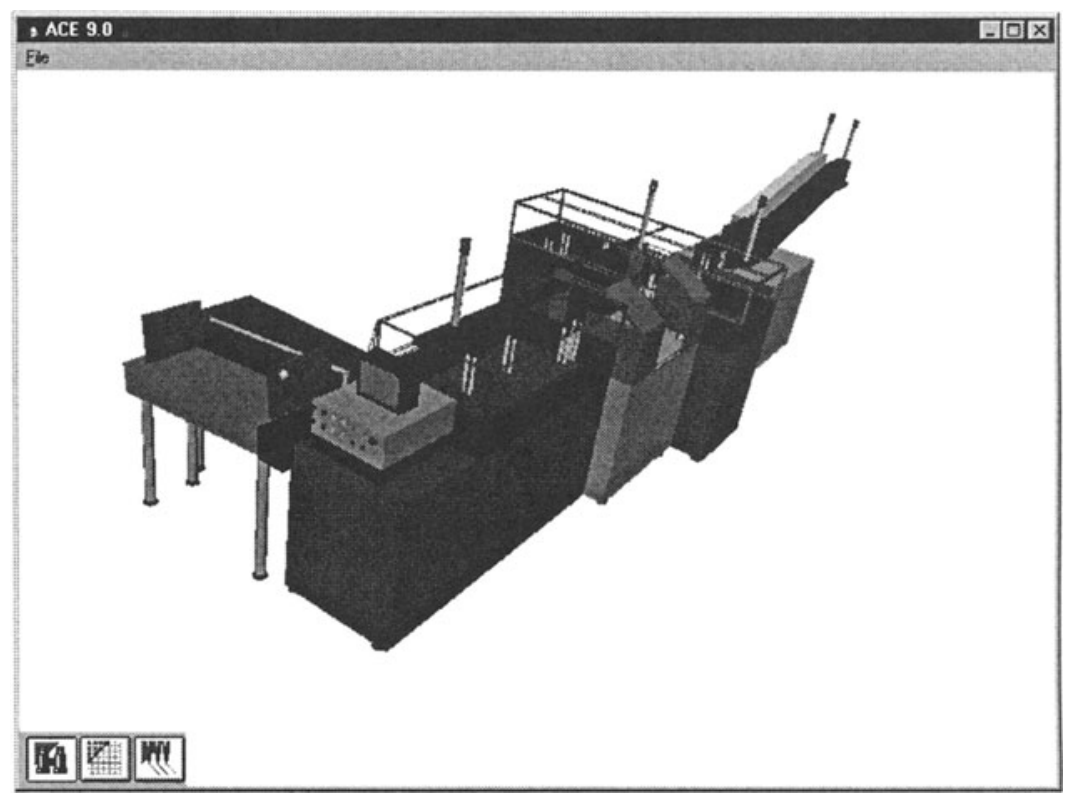

Figure 2. Automatic in-feed station (provided by the simulator)

In this example: - the automatic in-feed is done by a high-speed flatfeeder, HSFF, Fig. 2, via the in-feed magazine, the pick-off device and the double control-unit; - the processing is performed by an image lifter module, ILM, via the size measuring-unit, the bar code reader and the optical character recognition unit, and a the sorting and dispatching equipment, via 
the flat delay line, FDL, the label application module, LAM, the indexing module, IM and the sorter interface module, SIM; - the output delivery, finally, leads to the items boxing, via the diverting/forwarding devices, to the chosen tray/bin/bag out-feed units, or to the local reject module. The generic facility builds up as compact flat sorter module, CFSM, with main duties, Fig. 3; played by the in-feed buffer/sorter, IBS, the optical character recognition, OCR, device and the transport/singling system.

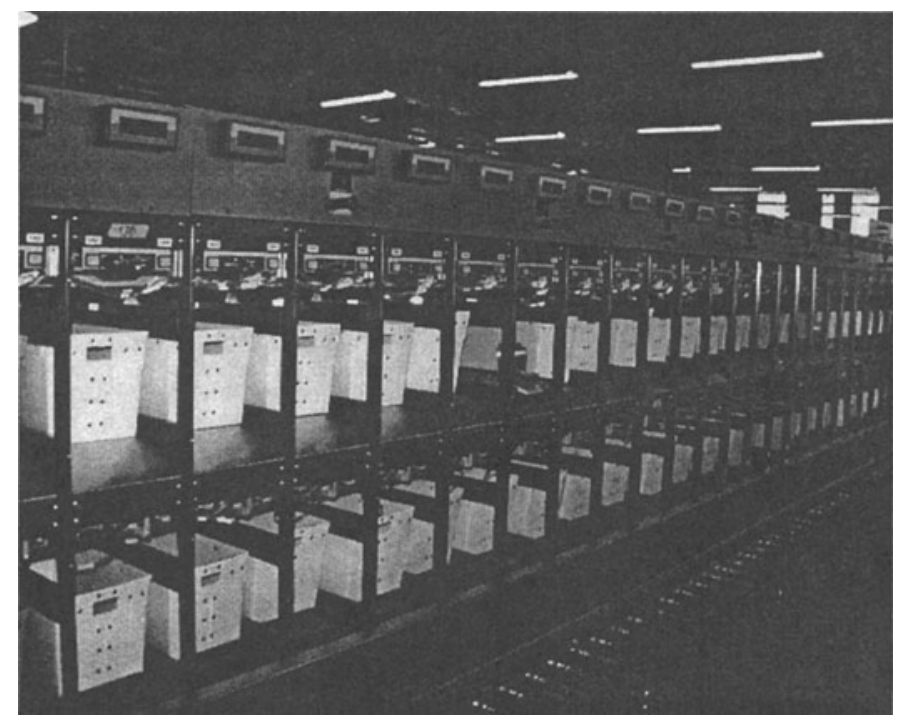

Figure 3. Example output delivery station

The operators are critically engaged at the sorting stands, where manual/automatic in-feed is scheduled, at the equipment up-keeping, for jam removal, and at the output section, to help forwarding the selected piled-up items. For the present study, focus is on the feeding stands, where, basically, three operator types distinguish: - ot, assigned to transfer the input trays; $o p$, involved at superintending the automatic portage; - om, employed for the manual feeding. The individual work-cycles are properly characterised as for timing and required energy, Fig. 4, with staffing depending on work-loads. The inclusion of on-process men provides simple supplying means for the automatic tracks, with the enhanced versatility of enabling manual by-passes when out of standard items shall be processed.

\subsection{The Operation Model and Task Scheduling}

To assess the proper outfits, alternative lay-outs have to be compared for actual working conditions. Resort to virtual prototyping and simulation runs 
makes these checks possible for wide operation spectra; to that goal, the AutoMod environment (by AutoSimulations Inc.) is used, due to the related graphic editor, to sketch animated plant units (machines, operators, transfer units, etc.) and the governing blocks, to enable the tasks evolution with recording of actual achievements. The 3D models assure a realistic view of the work-places and make easy to acknowledge the ergonomic figures of different duty sequences (as for operators' postures or efforts) and surroundings settings (as for noise, temperature, light, etc.), according to (off-line specified) job-allotment paradigms.

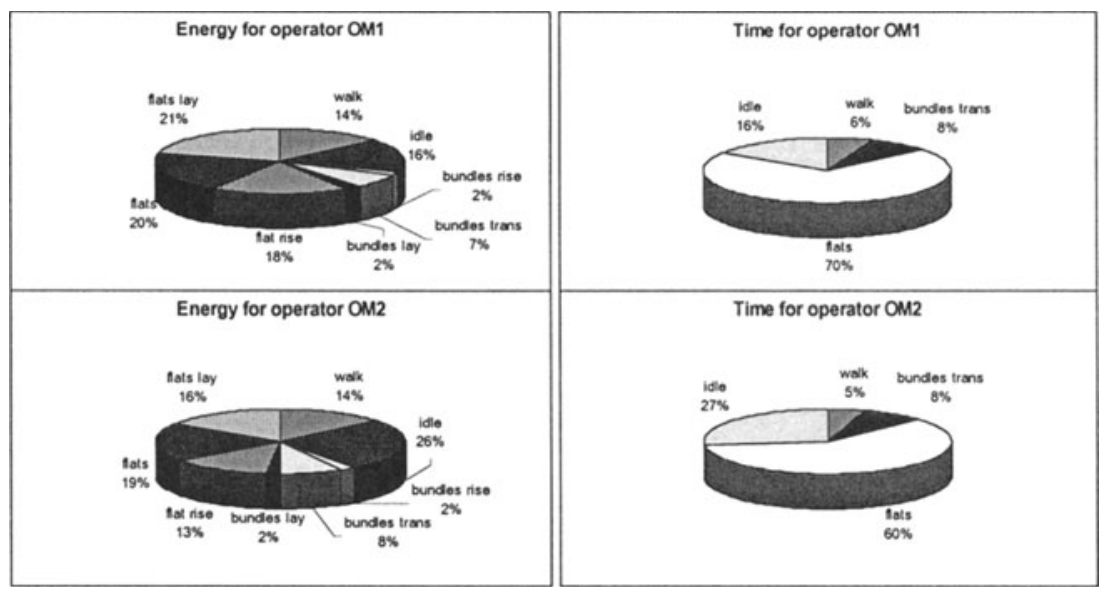

Figure 4. Example duty assignment (of the om operators)

Looking at the functional specification of a sorting and forwarding stand, the working out of the model implies to specify, and to encode as 'objects' (with properties and methods), all the physical and logic resources, relevant for describing the work-flow. Three object classes distinguish:

- machines: automatic in-feed module, manual in-feed module, sorter module, etc.;

- operators: ot (red), op (green), om (yellow);

- processed material: mix of flat items and related portage out-fits;

then a lay-out establishes, Fig. 5, with, e.g., two manual and three automatic in-feed units, the sorter module, the proper distribution of operators and the set of auxiliary facilities (trolleys with full/empty containers, tables with temporary mails stacks, etc.).

The generic feeding duty needs consider:

- the set of shuttles, carts or trolleys, each one carrying several containers (trays), with inside loose or bundled flats;

- the containers, with various mixes of loose flats or bundles of known amount of flats assorted by size; 
- the bundles, with series of journals or magazines, large wrappers, standard envelopes, etc.;

- the (out-of-standard) flats, loose or different thickness/length/height items.

The ot operator moves one tray at a time from the cart to a table, leaves the contents there and brings the empty container back; from their buffer table, the op operators supply an automatic in-feed unit and look after jams removal, as case arises; similarly, from their buffer table, the om operators recognise the flats, encode the address and manually in-feed the items toward the sorting line.

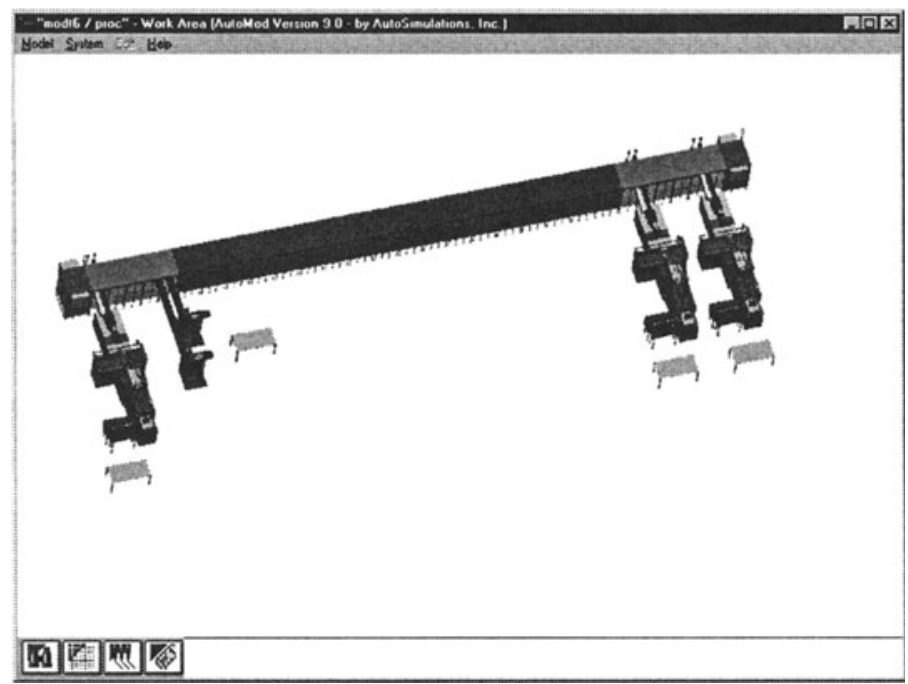

Figure 5. Virtual lay-out of the simulated plant

\subsection{The Reference Ergonomic Requirements}

The ergonomic checks deals with the relations: - humans vs. scheduled activity; - humans vs. machines; - humans vs. processed materials; - humans vs. surroundings. Proper choices are fixed by standards, such as, e.g., the ones, in the USA, of NIOSH, National Institute for Occupational Safety and Health. The physical activity is weighed by a labour index, ratio of the handled load and a 'recommended weight limit'; this is analysed into a series of factors, say: the actual weight, the vertical and horizontal location of the operator's hands by respect of his feet, the load displacement, the path off-set out of front manoeuvres, the operation frequency and the type of grasping device. The factors are ranged into classes and modified by figures, as for duty severity; this labour index should not exceed a given threshold; when 
an activity splits into several subtasks, each needs be weighed and shall not exceed the threshold.

The analysis leads to evaluate the 'ergonomic stress index', ESI, once fulfilled the scientific job-allotment description. Thereafter, the overall assigned duty-cycle is assessed in terms of metabolic energy expenditure, distinguishing five levels, from rest to very severe conditions and computing final estimates, with account of different operators and surroundings. During the design steps, the estimates combine the subtasks, to obtain the duty-cycle average figure. Experimental tests shall, generally, be ran on real set-ups, measuring the operators' oxygen consumption into actual work conditions, with: partial checks, covering the activity periods only, for light duties; or integral checks, covering as well the rest periods, for heavy duties.

The man/machine relations ought be verified as for the passive and active protection rules and as for the psycho-physic stress levels. The re-design of the work set-ups makes possible to rise the effectiveness, suggested by the habits of trained operators. The preparation of the material to be handled has relevant impacts: too huge or too heavy trays shell be avoided; for assorted items, the pre-processing of bundles mix enhances efficiency. Finally, the work-place benefits from properly fit lay-outs: for the in/out path, the light direction, the attention preservation, etc.; further requirements deals with health and safety prescriptions.

\section{MODELLING AND SIMULATION CHECKS}

The choice of the mail processing fixtures requires checks on the technical soundness and on the economical effectiveness; the former looks at ergonomic settings, the latter aims at fully exploiting the enabled resources. These checks start at the early ideation phases and need be accomplished for actual running conditions; resort to digital mock-ups makes easy to verify the achieved performance for the pertinent operation environments. By virtual testing, different lay-outs, obtained combining the basic modules and alternative auxiliary teams, are compared: during the design steps, to find out the technically optimised solution; during the operation steps, to balance the subsidiary man-made jobs within the work-flow.

The simulation snapshots aim at the realistic restitution of the surroundings, at least, so forth as items fetch, handling and in-feed are considered. The AutoMod (by AutoSimulations Inc.) supplies comparatively simple 3D displays of the basic modules, such as, for instance, the in-feed station, Fig. 6, the sorter, Fig. 7, etc., so that the finally assembled work space can be tested with actual running operation conditions. The description of a typical arrangement, Fig. 5, leads to the selected modules, with the related input queues (generated by the arrivals of items to be processed) and the assigned operators, with the subsidiary carts and tables; at the interface, 
physical and logical entities distinguish:

- the former details the current loads, e.g.: carrying-trays (with related attributes: transfer tasks, bundles identifiers, flats sizes and mixes, etc.); jam-occurrences (an initialiser randomly affects the individual items, to generate the drawback); jam-localisers (red/yellow lights show where jams occur); reset-flags (to identify actual lay-outs); cart-flags (to specify the status); ot-, op-, om- operators-labels (with attached on-going actions, say: transfer tasks from/to carts, tables, etc.; ancillary tasks for in-feed, jam removal, etc.; manual tasks for fetching and feeding, etc.); processflags (to rule items dispatch and sort flow, included jam generation occurrences);

- the latter deals with the task inventory and data transfer, e.g.: loads lists (for trays: cart/table fetch/delivery queues; for bundles: automatic/manual in-feed queues; for flats: hierarchy for automatic/manual entry and wait queues; for jams: delay to removal actions; for operators: delays before subsequent tasks); state variables (the occurrence-driven processes use loads to look into the scheduling list and reach the end-of-task when all actions are fulfilled).

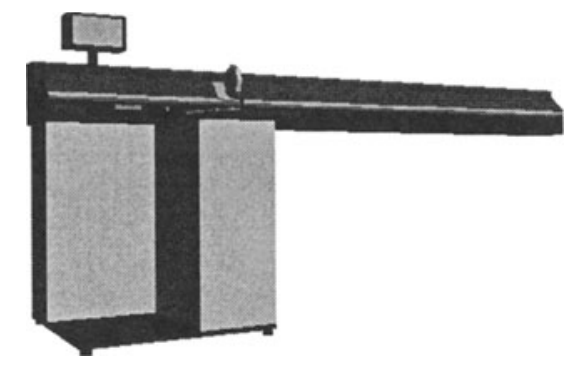

Figure 6. In-feed station for the virtual testing

\subsection{The Govern Logic and Simulation Setting}

The AutoMod code, further to realistic restitution capabilities, provides effective means to govern the facility time behaviour, combining queues and branches rules with functions and routines algorithms. The acknowledged loads have, actually, embedded attributes, so that task progression is directed by the chosen schedules; the simulation, however, profits by referring to loads managers, supplying explicit specification of the process govern. Few hints follow.

Several operators are concurrently engaged; their work-cycle is analysed to single out run-chains and cross-links and loads managers are encoded to 
synchronise the actions by message-passing queues: every operator has own jobs agenda, loads queue and wait lists. The govern unit of the ot-operator, for instance, deals with the work-cycle: - to pick up a full tray from a cart; to bring and place the tray on a table; - to empty the tray; - to pick up the empty tray; - to bring back the tray to a cart. The cycle repeats until all trays are processed. The task starts by means of two routines: cart assignment and table search; during the execution, the loads queue can modify the mail runchain or establish new cross-links; the cart search routine ends the cycle and is re-ran once a cart is completed. Similar analyses establish for every operator, looking at proper routines (for instance: for the op-, jam overseeing and removal; for the om- , flats singling out, reading and coding) and enabling the check and branch assignments.

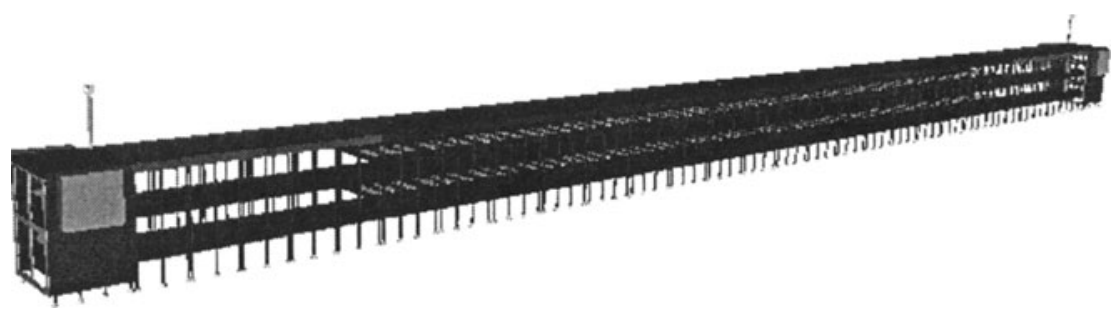

Figure 7. Sorter station for the virtual testing

The loads managers associated with $\boldsymbol{o t}$-, $\boldsymbol{o p}$-, $\boldsymbol{o m}$ - operators are re-set depending on resource allocation (number of men vs. carts, tables and stations) and efficiency patterns (superposed waviness and unexpected occurrences). Two further loads managers specify the govern rules of the automatic in-feed fixtures and, respectively, of the cart dispatching service, by means of the appropriate queues and wait lists and the related run-chains and cross-links.

\subsection{Example Simulation Studies and Assessments Issues}

Example developments are shortly summarised in the following for explanatory purpose. By simulation, the designer aims at verifying the technical appropriateness of the chosen sets of fixtures, the suitableness of given lay-outs and subsidiary resources, the plant effectiveness for the expected work-loads and the actually needed personnel depending on the throughput and on ergonomic figures. The study shall repeat in function of the facility characteristics, the work conditions, the operators' number and duties, etc. assessing, for given schedules, plant performance, resources' exploitation ratios, personnel stress and fatigue levels or other data on the reached issues. The digital mock-up is simply required to acknowledge the 
ergonomic features, to detect critical situations and to provide hints for better work conditions and plant efficiency, and AutoMod looks to be an adequate aid to that purpose, with effective restitution of the main functional and structural properties of the included resources, Fig. 8.

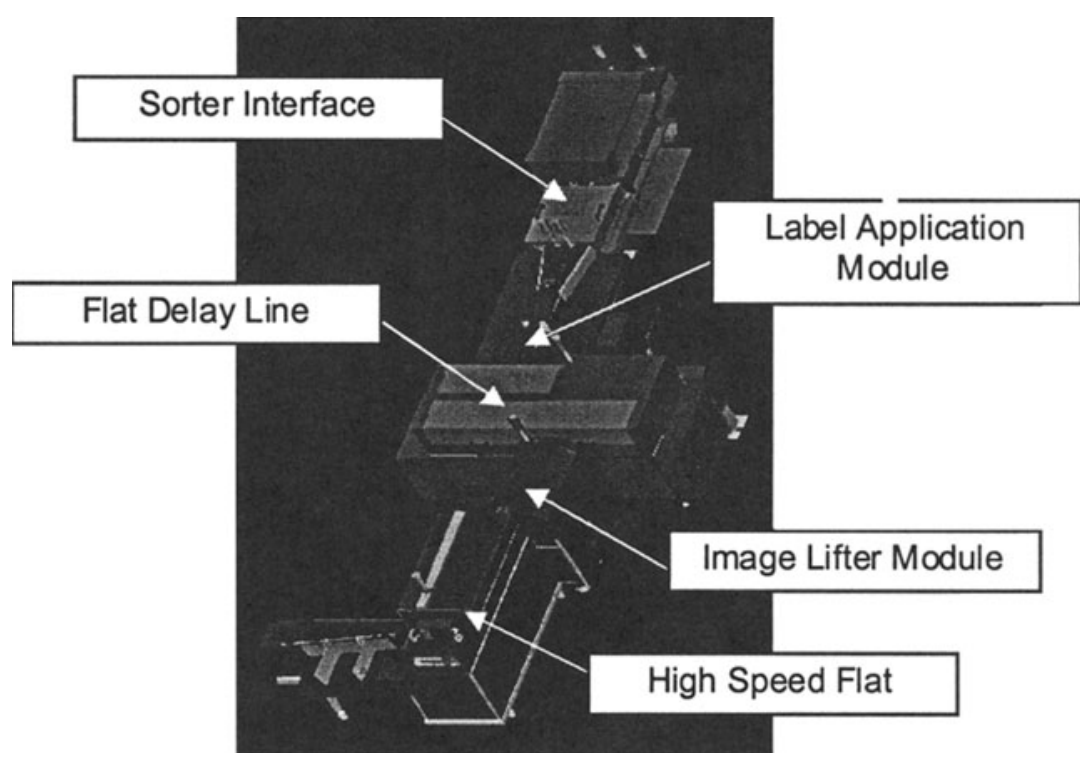

Figure 8. Functional and structural details of the simulated set-up

The generic simulation shall cover different work-shifts, each time with the resources (men and facilities) allocation depending on the loads agenda (e.g.: shifts, with high volume standard items; shifts, with low volumes special, as for size or weigh, items; etc.). The selected software does not offer a graphic page for re-defining each time the current lay-out; the drawback is removed by specifying a maximal configuration and declaring the sub-set of resources timely not included. Thereafter, at the input section two areas distinguish, for the automatic, either the manual in-feed; each area receives a set of carts (bearing five shelves with up to four trays each) and has a series of tables where the mail is temporarily placed. The ot-operators work between carts and tables; the op-operators feed the automatic stations (at the average rate: $0.5-0.6 \mathrm{~s} /$ flat); and the om-operators, the manual ones (at the average rate: 1.7-2 s/flat, including the reading/coding actions). The maximal configuration includes: up to three automatic and up to two manual in-feed stations; up to four ot- , three op- and two om-operators; the sorter unit has, therefore, to deal with that maximum throughput during the peak shifts.

To identify the operation characteristics, let refer to the main features, say: work-schedules, items yields and men commitment. For the scheduling, 
input data (single-index array) are the sets of the arrived full trays (and returned empty ones). The processed loads are described by double-index arrays: the first index, defining the affected operator; the second, the handled item (standard mail, packed bundle, special flat, etc.); each array is up-dated, as a new item is withdrawn from a tray and the pointer acknowledges the incumbency. This comes out from the duty analysis, namely, by assessing the time for trays handling, for items transportation, for mails manipulation, for flats in-feed, for jams removal, etc. including auxiliary actions (e. g., walking, taking position, and the likes).

The simulation data setting has to deal with:

- the agendas: lists of full trolleys (one for each of the enabled processing areas) and of related full trays, up-dated every time a new arrival is prompted;

- the operators' duties: amount of full/empty trays (in each area) on the trolleys/tables, up-dated every time a cycle is ended; amount of bundles/items ready for in-feed operations;

- the throughput: overall flats batches and mixes, given by two (one for the automatic, one for the manual in-feed) double-index arrays, to specify processing unit and items' type;

- the disturbances: jam occurrences, double-index array, to specify the line and the location;

- the labour indices: lists of actual actions (to stand by, walk, pickup/carry/lay-down a tray, take-out/lift/handle/place an item, acknowledge/encode a flat, recognise/remove/re-set a jam) and accounts of weighed figures.

Along with the daily work-shifts, the requests change, from early large amounts (e.g., due to the night mail piling-up), to variable steady conditions (e.g., depending on yearly seasons). The suitable mail sorter should grant the largest throughput and have sufficient buffering capacity in-between the infeed units so to avoid slowdown at bundles batch processing. The analysis aims at verifying the actual distribution of the operators during peak shifts and at assessing the manpower balance for typical steady runs, in terms of (average) duty-cycle times and of (standard) energy consumption and in front of the sought throughputs. The checks provide detailed views of the requested times and energies, showing whether critical situations arise or when proper balancing would improve the productivity; the results help the planning out of work-shifts on a day- or on a week-base, once the mails arrival is accordingly forecast. A set of example results will be orally presented and discussed, for explanatory purposes. Different processing strategies and resource allocation policies are compared to give evidence of situations that might approach critical issues. 


\section{CONCLUSIONS}

The life-cycle design of customer-driven artefacts or user-oriented fixtures is becoming winning skill, and resort to digital mock-up and computer simulation are effective means to immediately give factual assessments of the achieved performances. The paper is directly concerned by the ergonomic constraints of processing stands where operators and automatic machines are each other interfaced. The over-all effectiveness is highly affected by the agendas organisation and by the lay-out reflected conditions; the AutoMod models help the reaching of straightforward pictures for the on-progress plant behaviour and the simulation campaign readily supply the data to evaluate benefits and drawbacks of alternative setups, with direct measurement of the expected return on investment for any given mails sorting and forwarding stand. The tool deserves increasing relevance in the domain of assisted mailing machines, as competitiveness spreads on world-wide contexts and specialised services arise segmenting a market already deeply modified by the emerging information and communication technologies.

The paper first gives a bird's eye view of the assisted mails sorting and forwarding stands, based on integrated set-ups with operators, in-feed modules and mail sorting machines. The attention is turned to the masshandling of 'flat items' mixes with multiple-routing request, this being typical duty for universal postal utilities. The lay-out is obtained combining basic modules; the CAD tool is exploited, at the ideation stage, for the choice of the modules and the built up of the appropriate fit-outs, translating the all the all into an AutoMod code. The reference work-patterns requirements and related ergonomic prescriptions are, furthermore, considered to verify the effectiveness among competing set-ups, in terms of productivity and, both, to give evidence that the actions, actually selected for the duties fulfilment, lay within the labour protection acts, issued by the different national rules. These kind of tests is, on one side, required by the trade unions with due account of the social impact ranges; it is, the other side, critical reference to evaluate the return on investments.

The study, finally, refers to an example explanatory case, to compare competing lay-outs, accomplishing "flats" handling, sorting and dispatching operations, once the reference input mixes and output forwarding are given. The set-up includes manual and automatic stations and the simulation covers different options as for schedules, task engagement and work cycles. The issues give evidence of possible inconsistencies and hints on how to modify the lay-out to improve the productivity, while keeping optimal workconditions. 